\title{
Soroprevalência da infecção pelo vírus da hepatite B e pelo plasmódio em Lábrea, Amazonas: estimativa da ocorrência de prováveis coinfecções
}

\author{
Seroprevalence of hepatitis B and malaria infection in Lábrea, \\ Brazilian western Amazon: estimates of coinfection rates
}

\author{
Wornei Silva Miranda Braga ${ }^{1}$, Eva Batista da Silva ${ }^{1}$, Rita Auxiliadora \\ Botelho de Souza $^{1}$ e Carlos Eduardo Tosta ${ }^{2}$
}

\begin{abstract}
RESUMO
A Amazônia é conhecida pela elevada ocorrência de hepatite B e suas seqüelas. Contribui também com mais de 98\% dos casos de malária do país. Apesar de controvérsias, é proposto que quando associadas ocorram alterações na história natural das duas patologias. Este estudo estima a prevalência de prováveis coinfecções em população geral de área endêmica de ambas infecções na Amazônia Brasileira. A taxa de portadores do AgHBs encontrada foi de 3,3\% (IC 95\% 2,1\% a 5,1\%,), e a do anti- $\mathrm{HBc}$ total 49,9\% (IC 95\% 45,9\% a 53,8\%). A prevalência de anticorpos contra antígenos do Plasmodium vivax e Plasmodium falciparum foi de 51,4\% (311/605) (IC 95\% 47,3\% a 55,4\%). Em relação à presença simultânea de anticorpos contra antígenos do Plasmodium vivax e Plasmodium falciparum com marcadores do VHB, 1,8\% (11/605) , (IC95\% 1,0\% a 3,3\%), apresentavam também o AgHBs, tendo estes em média 26 anos de idade ( $p<0,001)$. Este estudo aponta semelhanças na distribuição dessas enfermidades como, a ocorrência preferencialmente entre adulto jovens. Os eventos provavelmente ocorrem em momentos distintos. Mostra também diferenças como, o baixo risco de malária entre menores de quinze anos, onde $0 \mathrm{VHB}$ circula com moderada intensidade. As taxas de coinfecções são provavelmente menores que as de portadores do AgHBs, apresentando padrão heterogêneo em relação ao espectro clínico da infecção pelo VHB.
\end{abstract}

Palavras-chaves: Hepatite B. Malária. Coinfecção. Amazônia.

\begin{abstract}
The Amazon region is known for the high occurrence of hepatitis B virus ( HBV) infection, and accounts for more than $98 \%$ of malaria cases in Brazil. Despite the controversy, it has been proposed that when associated they may lead to important effects in the natural history of both infections. This study estimates the prevalence of coinfection within general population of an endemic region of $\mathrm{HBV}$ and malaria in the Brazilian Amazon. The prevalence of $\mathrm{HBsAg}$ was 3.3\% (95\% CI 2.1\%-5.1\%,) and total anti-HBc 49.9\% (95\% CI 45.9\%-53.8\%). The prevalence of antibodies against Plasmodium vivax and Plasmodium falciparum antigens was 51.4\% (311/605) (95\% CI 47.3\%-55.4\%). Related to the simultaneous presence of malaria antibodies and HBV serological markers, in 1.8\% (11/605), (95\% CI 1.0\%-3.3\%), the presence of HBsAg was also demonstrated, mean age 26 years $(p<0.001)$. This study points to similarities in the distribution of these diseases, such as the occurrence mainly among young adults. The events may occur in different times. Also shown differences such as the low risk of malaria in the group up to fifteen years, where HBV circulates with moderate intensity. The prevalence of $\mathrm{HBV}$ and malaria coinfection is in fact less than the rates of $\mathrm{HBs} A g$ carriage, showing a heterogeneous pattern related to the clinical spectrum of $\mathrm{HBV}$ infection.
\end{abstract}

Key-words: Hepatitis B. Malaria. Coinfection. Amazon.

1. Fundação de Medicina Tropical do Amazonas, Manaus, AM. 2. Núcleo de Medicina Tropical e Nutrição da Universidade de Brasília, Brasília, DF. Endereço para correspondência: Dr. Wornei Braga. Gerência de Virologia/FMTAM. Av. Pedro Teixeira 25, D. Pedro I, 69040-000 Manaus, AM. Fax: 5592 238-3762.

e-mail: imtam@ prodamnet.com.br; wornei@ hotmail.com

Recebido para publicação em 3/2/2004

Aceito em 2/3/2005 
A infecção pelo vírus da hepatite B (VHB) e pelo plasmódio são muito comuns na Amazônia, onde a ocorrência de coinfecções é tida também como supostamente muito freqüente.

A associação entre 0 VHB e 0 plasmódio foi estudada em modelos experimentais, tendo sido demonstrado gradiente de suscetibilidade à infecção pelo VHB em macacos Rhesusinfectados crônicamente pelo Plasmodium inuii ${ }^{20} \mathrm{e}$ é descrita a inibição da replicação do VHB, em camundongos transgênicos, infectados pelo Plasmodium yoelii ${ }^{19}$.

Em relação a essa associação em humanos, os resultados são controversos. № Brasil, é descrito maior comprometimento hepático, presença de esplenomegalia e persistência mais prolongada de anticorpos antiplasmódio na coinfecção entre 0 VHB e malária por Plasmodium vivax?.

Na Gâmbia ${ }^{22}$, e no Vietnam ${ }^{1}$, a presença do AgHBs foi implicada como fator de risco para desenvolver quadro grave de malária. Em Papua-Nova Guiné, apesar de elevadas taxas de portadores do AgHBs entre indivíduos de origem Australóide, as maiores taxas de hiperesplenismo foram encontradas entre indivíduos de outras etnias, em que as taxas de prevalência do VHB encontradas foram menores4. Também, não foi encontrada associação entre a presença do VHB e elevadas parasitemias ${ }^{5}$.

As regiões do planeta de maior ocorrência de malária e de hepatite B são justapostas, no entanto, pouco tem sido produzido em relação a associação dos dois agentes.

0 presente estudo tem por objetivo estimar a prevalência de marcadores sorológicos de infecção presente e passada do VHB, de marcadores sorológicos de infecção pelo Plasmodium falciparum ou Plasmodium vivax, e da associação dos marcadores sorológicos das duas infecções, estimando o impacto da presença de uma infecção sobre a prevalência da outra, avaliando também possiveis fatores de risco para ambas infecções.

\section{MATERIAL E MÉTODOS}

Este é um estudo de prevalência, de base populacional, desenvolvido no município de Lábrea, estado do Amazonas, bacia do rio Purus, tendo como população alvo os moradores da zona urbana.

Considerando que a prevalência de coinfecção malária e VHB é desconhecida, tomou-se como base para 0 cálculo do tamanho da amostra, a população da cidade de Lábrea, 18.000 habitantes (estimativa da Secretaria de Estado da Saúde do Amazonas - 1999) e uma prevalência estimada de portadores do AgHBs de 7\% ${ }^{6}$. 0 programa Epi-Info ${ }^{10}$ foi utilizado para calcular 0 tamanho da amostra, através do módulo Epitable calculator, que deu uma amostra mínima de 548 indivíduos necessários para a investigação.

A seleção dos indivíduos se deu por sorteio das residências por amostragem aleatória simples. Dos indivíduos selecionados e autorizado a participação, foi preenchido um questionário e coletado $10 \mathrm{ml}$ de sangue, por punção venosa com sistema vacutainer, e punção digital para realização do teste da gota espessa.

Para os testes sorológicos do VHB (pesquisa do AgHBs e do anti-HBc total) foi empregado o método imunoenzimático
(ELISA), utilizando-se kits comerciais (Organon Teknica ${ }^{\circledR}$ ), segundo orientações do fabricante. 0 anti-HBs não foi testado, pois identificar indivíduos com marcador de proteção contra 0 VHB por vacinação (anti-HBs isolado) não foi objetivo específico da pesquisa, estes na verdade estão incluídos entre os só com marcador de malária ou entre os sem marcador do VHB ou de malária, usados como controles para indivíduos com marcadores de ambas infecções. Todas as amostras foram também testadas (teste qualitativo) para anticorpos de classe IgM e IgG contra P. vivax ou P. falciparum, pelo método imunoenzimático (ELISA) empregando-se antígenos obtidos no laboratório da gerência de malária da FMTAM.

As informações foram registradas em um banco de dados, e análises estatísticas conduzidas utilizando-se 0 Epi-Info $6.04^{10}$. A presença de exposição a fatores de risco foi dicotomizada em presente ou ausente, para determinar se existia associação entre a exposição ao fator e a presença de marcador sorológico de hepatite ou malária, medindo-se a associação através do cálculo das razões de prevalência ( $R P)$.

0 protocolo foi autorizado pelo Comitê de Ética em Pesquisa da FMT/IMT-AM e seguiu as determinações definidas na resolução 196/96 do Conselho Nacional de Saúde do Ministério da Saúde, os participantes foram informados dos objetivos, vantagens e possiveis riscos dos procedimentos, sendo sua participação autorizada mediante assinatura do termo de consentimento, em caso de indivíduo menor de idade o consentimento era dado pelo responsável presente na hora da entrevista.

\section{RESULTAD0S}

0 estudo incluiu 605 indivíduos pertencentes a um total de 125 famílias. A taxa de portadores de AgHBs encontrada foi 3,3\% ( Tabela 1). Presente em 2,7\% dos indivíduos entre cinco e 14 anos; 5,7\% entre os de 15 e 29 anos e 6,1\% entre os de 30 e 50 anos de idade, diferença estatisticamente significativa ( $X^{2}=11,14 ; \mathrm{GL}=5, \mathrm{p}=0,04$; Tabela 2 ).

0 AgHBs mostrou associação estatística significativa com passado de hepatite, (razão de prevalência de 6,59; IC-95\% 2,7-15,7; $p<0,001$, teste exato de Fisher) e níveis elevados de alaninoaminotransferase (ALT) ( $R P=9,41$; IC- $95 \%$ 2,68-33,1; $p=0,02$, teste exato de Fisher (Tabela 3).

A taxa de prevalência do marcador de contato com o VHB (antiHBc total isolado) encontrada foi $49,9 \%$, Tabela 1, apresentando gradiente crescente em relação à idade, chegando a níveis bastante elevados ( $80 \%$ ) em indivíduos com mais de 50 anos ( $\mathrm{X}^{2}$ para tendência igual a 191,025; p<0,001; Tabela 2). A presença deste marcador mostrou-se associada à história de hepatite $(\mathrm{RP}=1,31$; IC-95\% 1,08-1,58; $p=0,01$ ), indivíduos nascidos na zona rural do município, razão de prevalência de 2,44 (IC-95\% 1,99-2,99; $\mathrm{p}<0,001)$ e história de vacinação contra hepatite $\mathrm{B}$, razão de prevalência de 0,78 (IC-95\% 0,65-0,94; p= 0,01) ( Tabela 3).

Apenas três indivíduos apresentaram anticorpo da classe IgM contra o P. vivax e, nenhuma das amostras avaliadas apresentou reatividade para 0 anticorpo da classe IgM contra 0 P. falciparum. 
Tabela 1 - Prevalência de marcadores sorológicos doVHB e infecção por plasmódio segundo procedência, Lábrea, Amazonas, 2000.

\begin{tabular}{|c|c|c|c|c|c|c|c|c|c|c|c|}
\hline \multirow[b]{2}{*}{ Local de nascimento } & \multicolumn{2}{|c|}{ Amostra } & \multicolumn{3}{|c|}{ AgHBs reativo } & \multicolumn{3}{|c|}{ Anti-HBc total reativo } & \multicolumn{3}{|c|}{$\begin{array}{l}\text { Anticorpos contra P. vivax } \\
\text { ou P. falciparum }{ }^{c}\end{array}$} \\
\hline & $\mathrm{n}^{0}$ & $\%^{\mathrm{a}}$ & $\mathrm{n}^{0}$ & $\%^{\mathrm{b}}$ & IC 95\% & $\mathrm{n}^{0}$ & $\%^{b}$ & IC $95 \%$ & $\mathrm{n}^{0}$ & $\%^{b}$ & IC95\% \\
\hline Lábrea & 283 & 46,8 & 7 & 2,5 & $0,7-4,3$ & 82 & 29,0 & $23,8-34,2$ & 100 & 35,3 & $29,8-40,8$ \\
\hline Zona rural & 312 & 51,6 & 12 & 3,8 & $1,7-5,9$ & 215 & 68,9 & $63,8-74,0$ & 212 & 67,9 & $62,8-73,0$ \\
\hline Outras regiões & 10 & 1,7 & 1 & 10,0 & $0,0-28,5$ & 5 & 50,0 & $19,1-80,9$ & 4 & 40,0 & $9,7-70,3$ \\
\hline Total & 605 & 100,0 & 20 & 3,3 & $1,9-4,7$ & 302 & 49,9 & $45,9-53,8$ & 311 & 51,4 & $47,3-55,4$ \\
\hline
\end{tabular}

AgHBs, antígeno de superfície do vírus da hepatite B (VHB); anti-HBc total, anticorpo, de classe IgG, contra o antígeno do core do VHB; IC-95\%, intervalo de confiança;

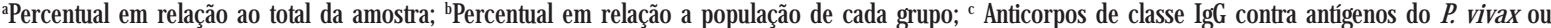
P. falciparum

Tabela 2 - Prevalência de marcadores sorológicos do VHB, infecção pelo plasmódio e presença simultânea com o AgHBs por grupo de idade, Lábrea, Amazonas, 2000.

\begin{tabular}{|c|c|c|c|c|c|c|c|c|c|c|c|c|c|c|c|c|}
\hline \multirow[t]{2}{*}{ Idade } & \multicolumn{2}{|c|}{ Amostras } & \multicolumn{3}{|c|}{ AgHBs reativo } & \multicolumn{2}{|c|}{ AgHBe reativo } & \multicolumn{3}{|c|}{$\begin{array}{l}\text { Anti- } \mathrm{HBC} \\
\text { total reativo }\end{array}$} & \multicolumn{3}{|c|}{$\begin{array}{l}\text { Anticorpos contra } \\
\text { P. vivax ou P. falciparum }\end{array}$} & \multicolumn{3}{|c|}{$\begin{array}{l}\text { Anticorpos contra P. vivax ou } \\
\text { P. falciparum }{ }^{c} \text { com a presença do } \mathrm{Ag} H \mathrm{HB}\end{array}$} \\
\hline & $\mathrm{n}^{0}$ & $\%$ & $\mathrm{n}^{0}$ & $\%^{\mathrm{a}}$ & IC-95\% & $\mathrm{n}^{0}$ & $\%^{\mathrm{b}}$ & $\mathrm{n}^{0}$ & $\%^{\mathrm{a}}$ & IC-95\% & $\mathrm{n}^{0}$ & $\%^{\mathrm{a}}$ & IC-95\% & $\mathrm{n}^{0}$ & $\%^{\mathrm{a}}$ & IC-95\% \\
\hline$<1$ & 18 & 3,0 & 0 & 0 & - & 0 & 0 & 0 & 0 & - & 4 & 22,2 & $3,0-41,4$ & 0 & 0 & - \\
\hline $2-4$ & 94 & 15,5 & 0 & 0 & - & 0 & 0 & 6 & 6,4 & $1,5-11,3$ & 30 & 31,9 & $22,5-41,3$ & 0 & 0 & - \\
\hline $5-14$ & 182 & 30,1 & 5 & 2,7 & $0,4-5,0$ & 2 & 10,0 & 49 & 26,9 & $20,5-27,3$ & 74 & 40,7 & $33,6-47,8$ & 3 & 1,6 & $-0,2-3,4$ \\
\hline $15-29$ & 158 & 26,1 & 9 & 5,7 & $2,1-9,3$ & 0 & 0 & 123 & 77,8 & $71,3-84,3$ & 92 & 58,2 & $50,6-65,8$ & 5 & 3,2 & $0,5-5,9$ \\
\hline $30-49$ & 98 & 16,2 & 6 & 6,1 & $1,4-10,8$ & 1 & 5,0 & 80 & 81,6 & $74,0-89,2$ & 68 & 69,4 & $60,3-78,5$ & 3 & 3,1 & $-0,3-6,5$ \\
\hline$\geq 50$ & 55 & 9,1 & 0 & 0 & - & 0 & 0 & 44 & 80,0 & $69,5-90,5$ & 43 & 78,2 & $67,3-89,1$ & 0 & 0 & - \\
\hline Total & 605 & 100,0 & 20 & 3,3 & $1,9-4,7$ & 3 & 15,0 & 302 & 49,9 & $45,9-53,8$ & 311 & 51,4 & $47,3-55,4$ & 11 & 1,8 & $1,0-3,3$ \\
\hline
\end{tabular}

AgHBs, antígeno de superfície do vírus da hepatite B; AgHBe, antígeno e do vírus da hepatie B; Anti-HBc total, anticorpo contra o antígeno do core do vírus da hepatite B; IC-95\%,

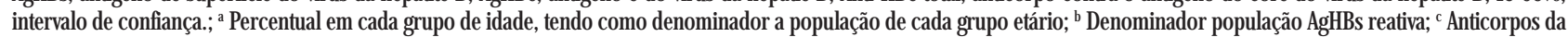
classe IgG contra antígenos do P. vivax ou P. falciparum.

Tabela 3 - Fatores associados à presença do AgHBs ou do anti-HBc total isolado.

\begin{tabular}{|c|c|c|c|c|c|c|c|c|c|c|c|c|c|c|}
\hline \multirow[t]{2}{*}{ Exposiçãa } & \multicolumn{2}{|c|}{$\begin{array}{c}\text { AgHBs reativo } \\
\text { na presença do fator }\end{array}$} & \multicolumn{5}{|c|}{$\begin{array}{c}\text { AgHBs reativo } \\
\text { sem a presença do fator }\end{array}$} & \multicolumn{2}{|c|}{$\begin{array}{l}\text { Anti-HBc total isolado } \\
\text { na presença do fator }\end{array}$} & \multicolumn{5}{|c|}{$\begin{array}{l}\text { Anti-HBc total isolado } \\
\text { sem presença do fator }\end{array}$} \\
\hline & $\mathrm{n}^{0}$ & $\%$ & $\mathrm{n}^{0}$ & $\%$ & $\mathrm{RP}$ & IC-95\% & $\mathrm{p}$ & $\mathrm{n}^{0}$ & $\%$ & $\mathrm{n}^{0}$ & $\%$ & $\mathrm{RP}$ & IC-95\% & $\mathrm{p}$ \\
\hline Vacina contra VHB* & $7 / 302$ & 2,3 & $12 / 284$ & 4,2 & 0,55 & $0,22-1,37$ & 0,28 & $76 / 295$ & 25,8 & $194 / 272$ & 71,3 & 0,78 & $0,65-0,94$ & 0,01 \\
\hline História de hepatite* & $12 / 112$ & 10,7 & $8 / 492$ & 1,6 & 6,59 & $2,76-15,7$ & $<0,001$ & $60 / 100$ & 60,0 & $222 / 484$ & 45,9 & 1,31 & $1,08-1,58$ & 0,01 \\
\hline Transfusão de sangue* & * $2 / 23$ & 8,7 & $18 / 580$ & 3,1 & 2,80 & $0,69-11,3$ & 0,17 & $17 / 21$ & 81,0 & $264 / 562$ & 47,0 & 1,04 & $0,83-1,29$ & 0,99 \\
\hline História de malária & $9 / 250$ & 3,6 & $11 / 352$ & 3,1 & 1,15 & $0,48-2,74$ & 0,92 & $160 / 241$ & 66,4 & $119 / 341$ & 34,9 & 1,11 & $0,97-1,26$ & 0,18 \\
\hline ALT elevada & $2 / 7$ & 28,6 & $18 / 593$ & 3,0 & 9,41 & $2,68-33,1$ & 0,02 & 4/279 & 1,4 & $1 / 301$ & 0,3 & 4,32 & $0,49-38,4$ & 0,20 \\
\hline Zona rural ${ }^{\mathrm{a}}$ & $12 / 312$ & 3,8 & $8 / 293$ & 2,7 & 1,44 & $0,58-3,40$ & 0,58 & $203 / 300$ & 67,7 & $79 / 285$ & 27,7 & 2,44 & $1,99-2,99$ & $<0,001$ \\
\hline
\end{tabular}

AgHBs, antígeno de superfície do VHB; anti-HBc total, anticorpo contra o antígeno do core do VHB; RP, Razão de prevalência; IC95\%, intervalo de confiança; p, significância estatística. a. Indivíduos nascidos na zona rural de município; * Ajustado por idade.

Ataxa de prevalência de portadores de anticorpos da classe IgG contra o P. vivax ou P. falciparum encontrada foi 51,4\%, variando de 35,3\% entre os indivíduos nascidos na zona urbana de Lábrea a $67,9 \%$ entre os procedentes da zona rural do município $\left(\mathrm{X}^{2}=55,88\right.$; $\mathrm{GL}=1 ; \mathrm{p}<0,001$; Tabela 1). Apresentando gradiente crescente em relação a idade ( $X^{2}$ para tendência igual a 66,242; $p<0,001$ ).

Năo observamos associação estatisticamente significativa entre a presença de anticorpos contra antígenos de P. vivax ou P. falciparum e sexo, história passada de hepatite, hepatite na família, vacinação contra hepatite B e níveis séricos de ALT, estando fortemente associada à história pregressa de malária $(\mathrm{RP}=4,59$; IC-95\% 3,73-5,64; $\mathrm{p}<0,001)$ e local de nascimento (indivíduos nascidos na zona rural do município; $\mathrm{RP}=1,88$; IC-95\% 1,57-2,24; $p<0,001$; Tabela 4).

Em relação à presença simultânea de anticorpos contra antígenos do P. vivax ou P. falciparum e marcadores do VHB, 1,8\% dos indivíduos, apresentaram marcadores de malária e 0 AgHBs. A presença simultânea com 0 anti-HBc total foi encontrada em 32,9\% dos indivíduos estudados, Tabela 2.
Os indivíduos anti-HBc total reativos apresentaram uma prevalência cerca de duas vezes maior de infecção passada pelo P. falciparum ou P. vivax ( $\mathrm{RP}=1,78$; IC-95\% 1,51-2,11; $\mathrm{p}<0,001)$. Após estratificação por grupo de idade, a magnitude dessa associação apresenta variação em relação aos grupos etários ( teste para homogeneidade $\mathrm{X}^{2}=15,92$ $\mathrm{p}=0,003)$, mantendo-se significativa nos indivíduos de cinco a quinze anos ( $\mathrm{RP}=1,56$; $\mathrm{IC}-95 \%$ 1,11-2,19; $\mathrm{p}=0,01)$ e entre os de quinze a trinta anos ( $\mathrm{RP}=1,90$; IC- $95 \% 1,18-3,06$; $\mathrm{p}=0,001$; Tabela 5).

No presente estudo, não foi observado associação estatisticamente significativa entre a presença simultânea de anticorpos contra antígenos do P. vivax ou P. falciparum com o AgHBs ou anti-HBc total, história passada de hepatite, vacinação contra hepatite $\mathrm{B}$ e níveis séricos de $\mathrm{ALT}$, estando associado à história pregressa de malária ( $\mathrm{RP}=6,60$; IC-95\% 4,76-9,15; $\mathrm{p}<0,001$ ) e local de nascimento (indivíduos nascidos na zona rural do município) ( $\mathrm{RP}=2,87$; IC-95\% 2,173,81; $\mathrm{p}<0,001$; Tabela 6). 
Tabela 4 - Fatores associados à presença de marcadores sorológicos de infecção pelo plasmódio.

\begin{tabular}{|c|c|c|c|c|c|c|c|}
\hline \multirow{3}{*}{ Exposição } & \multirow{2}{*}{\multicolumn{2}{|c|}{$\begin{array}{l}\text { Anticorpos contra P. vivax ou } \\
\text { P. falciparum }{ }^{\mathrm{a}} \text { na a presença do fator }\end{array}$}} & \multirow{2}{*}{\multicolumn{2}{|c|}{$\begin{array}{l}\text { Anticorpos contra P. vivax ou } \\
\text { P. falciparum }{ }^{\mathrm{a}} \text { sem a presença do fator }\end{array}$}} & \multirow{3}{*}{$\mathrm{RP}$} & \multirow{3}{*}{ IC-95\% } & \multirow{3}{*}{$\mathrm{p}$} \\
\hline & & & & & & & \\
\hline & $\mathrm{n}^{0}$ & $\%$ & $\mathrm{n}^{0}$ & $\%$ & & & \\
\hline Vacina contra VHB* & $124 / 302$ & 41,1 & $176 / 284$ & 62,0 & 1,02 & $0,83-1,24$ & 0,97 \\
\hline História de hepatite* & $60 / 112$ & 53,6 & $251 / 492$ & 51,0 & 1,09 & $0,78-1,52$ & 0,70 \\
\hline Transfusão de sangue* & $16 / 23$ & 69,6 & $294 / 580$ & 50,7 & 2,16 & $0,90-5,18$ & 0,11 \\
\hline História de malária & $238 / 250$ & 95,2 & $73 / 352$ & 20,7 & 4,59 & $3,73-5,64$ & $<0,001$ \\
\hline ALT elevada & $4 / 307$ & 1,3 & $3 / 293$ & 1,0 & 1,27 & $0,29-5,64$ & 0,75 \\
\hline Zona rural ${ }^{b}$ & 207/312 & 66,3 & $104 / 293$ & 35,5 & 1,88 & $1,57-2,24$ & $<0,001$ \\
\hline
\end{tabular}

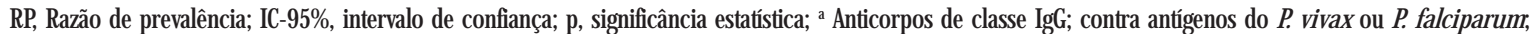
${ }^{\mathrm{b}}$ Indivíduos nascidos na zona rural do município. * Ajustado por idade.

\begin{tabular}{|c|c|c|c|c|c|c|c|}
\hline \multirow[b]{2}{*}{ Grupo de idade } & \multicolumn{2}{|c|}{$\begin{array}{l}\text { Anti-HBc total reativo } \\
\text { com marcador de malária }\end{array}$} & \multicolumn{2}{|c|}{$\begin{array}{l}\text { Anti-HBc total não reativo } \\
\text { com marcador de malária }\end{array}$} & \multirow[b]{2}{*}{$\mathrm{RP}^{\mathrm{c}}$} & \multirow[b]{2}{*}{ IC-95\% } & \multirow[b]{2}{*}{$\mathrm{p}$} \\
\hline & $\mathrm{n}^{\text {oa }}$ & $\%^{\mathrm{b}}$ & $\mathrm{n}^{\mathrm{oa}}$ & $\%^{\mathrm{b}}$ & & & \\
\hline$<1$ & - & - & - & - & - & $\cdot$ & \\
\hline $1-4$ & $3 / 6$ & 50,0 & $27 / 88$ & 30,7 & 1,63 & $0,69-3,85$ & 0,33 \\
\hline $5-14$ & $27 / 49$ & 55,1 & $47 / 133$ & 35,3 & 1,56 & $1,11-2,19$ & 0,01 \\
\hline $15-29$ & $80 / 123$ & 50,1 & $12 / 35$ & 34,3 & 1,90 & $1,18-3,06$ & 0,001 \\
\hline $30-49$ & $53 / 80$ & 66,3 & $15 / 18$ & 83,3 & 0,80 & $0,61-1,03$ & 0,15 \\
\hline$\geq 50$ & $36 / 44$ & 81.8 & $7 / 11$ & 63,6 & 1,29 & 0,81-2,05 & 0,19 \\
\hline Total & 199/302 & 65,9 & $112 / 303$ & 37,0 & 1,78 & 1,51-2,11 & $<0,001$ \\
\hline
\end{tabular}

RP, Razão de prevalência; IC-95\%, Intervalo de confiança; P, Probabilidade da hipótese nula ser verdadeira; a. Frequência absoluta em cada grupo; b. Prevalência em cada grupo; c. RP para cada grupo; RP ajustada após estratificação por idade $(\mathrm{RP}=1,38, \mathrm{IC}-95 \% 1,14-1,67)$. Teste de homogeneidade $\mathrm{X} 2=15,92, \mathrm{p}=0,003$.

Tabela 6 - Fatores associados à presença simultânea de marcadores sorológicos de infecção pelo plasmódio e do VHB*.

\begin{tabular}{|c|c|c|c|c|c|c|c|}
\hline \multirow[b]{2}{*}{ Exposição } & \multicolumn{2}{|c|}{$\begin{array}{l}\text { Anticorpos contra P. vivax } \\
\text { ou P. falciparuma com marcadores } \\
\text { do VHB* na presença do fator }\end{array}$} & \multicolumn{2}{|c|}{$\begin{array}{l}\text { Anticorpos contra P. vivax do VHB* } \\
\text { ou P. falciparum }{ }^{\mathrm{a}} \text { com marcadores } \\
\text { sem a presença do fator }\end{array}$} & \multirow[b]{2}{*}{$\mathrm{RP}$} & \multirow[b]{2}{*}{ IC-95\% } & \multirow[b]{2}{*}{$p$} \\
\hline & $\mathrm{n}^{0}$ & $\%$ & $\mathrm{n}^{0}$ & $\%$ & & & \\
\hline Vacina contra VHB** & $51 / 302$ & 16,9 & $140 / 284$ & 49,3 & 0,85 & $0,64-1,13$ & 0,31 \\
\hline História de hepatite** & $44 / 112$ & 39,3 & $155 / 492$ & 31,5 & 1,25 & $0,96-1,62$ & 0,14 \\
\hline Transfusão** & $13 / 23$ & 56,5 & $185 / 588$ & 31,9 & 1,03 & $0,71-1,50$ & 0,95 \\
\hline História de malária & $164 / 250$ & 65,6 & $35 / 352$ & 9,9 & 6,62 & $4,76-9,15$ & $<0,001$ \\
\hline ALT elevada & $4 / 7$ & 57,1 & $192 / 593$ & 32,4 & 1,76 & $0,92-3,39$ & 0,32 \\
\hline Zona rural ${ }^{b}$ & $150 / 312$ & 48,1 & $49 / 293$ & 16,7 & 2,87 & $2,17-3,81$ & $<0,001$ \\
\hline
\end{tabular}

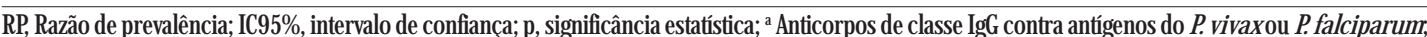

${ }^{\mathrm{b}}$ Indivíduos nascidos na zona rural do município; * AgHBs ou anti-HBc total isolado; ** Ajustado por idade.

\section{DISCUSSÃ0}

Este estudo foi capaz de estimar a prevalência de marcadores sorológicos de infecção por Plasmodium vivax e Plasmodium falciparum, pelo VHB e da presença simultânea de marcadores de ambas enfermidades, em população de uma área endêmica de malária e de hepatite B na Amazônia ocidental Brasileira.

A taxa de prevalência de portadores do AgHBs encontrada em Lábrea identifica um padrão de endemicidade moderada ${ }^{21}$, porém cerca de três vezes maior que a referida para a cidade de São Paulo ${ }^{11}$. Dois grupos parecem ser importantes na circulação do VHB nesta população, um de crianças entre cinco e 14 anos de idade e outro de indivíduos adultos jovens entre 15 e 29 anos de idade.
Como a transmissão vertical ou perinatal é descrita como de pouca importância na região Amazônica ${ }^{6}{ }^{12} 19$ e 0 estudo não identificou nenhuma grávida ou menor de cinco anos portador do AgHBs, os mecanismos de transmissão do VHB talvez estejam relacionados a fatores não identificados nesta pesquisa, provavelmente associados a aspectos ambientais ${ }^{218}$ ou socioculturais ${ }^{3}$. Diversos estudos experimentais e epidemiológicos indicam a possibilidade de transmissão do VHB por insetos hematófagos ${ }^{816}$, sugerindo que este tipo de transmissão contribua para a elevada endemicidade em regiões tropicais.

A distribuição da prevalência de marcador sorológico de contato com o VHB revela padrão de intensa circulação do vírus. Como a vacina contra hepatite B foi introduzida na região em 1989 e, os indivíduos acima de vinte anos, fazem parte da população que não foi exposta à vacina, e a transmissão parece 
ter sido interrompida em menores de cinco anos, espera-se somente um impacto, a longo prazo, no número de indivíduos infectados e de portadores crônicos nesta população.

Foi encontrada uma elevada prevalência de anticorpos contra antígenos do Plasmodium vivax ou Plasmodium falciparum na população estudada, com provável predominância de casos passados por malária vivax, padrão comum na Amazônia Brasileira' ${ }^{13} 14$. Apesar de ter sido encontrado em todas as idades, apresenta gradiente crescente em relação à idade. Como a presença de anticorpos da classe IgGcontra antígenos do plasmódio étida como indicador de exposição e não de imunidade protetora ${ }^{93}$, 0 padrão encontrado mostraria transmissão mais freqüente nos adultos, entretanto, existe dificuldade na interpretação dos resultados pela escassez de estudos que avaliem 0 comportamento de anticorpos contra 0 plasmódio a longo prazo e, nossos resultados sugerem que a presença desses marcadores possam ter longa duração.

0 risco da presença simultânea de marcador de contato com o VHB e de marcador sorológico de infecção passada por uma das espécies de plasmódio, observada nos indivíduos de cinco a trinta anos de idade, apresenta baixa magnitude com elevada significância estatística, sugerindo possível associação entre esses dois agentes permitindo que se levante as seguintes possibilidades: (1) que uma parcela significativa da população estudada vive em condições epidemiológicas que favoreçam a transmissão de ambas infecções, (2) que possam estar sendo transmitidos por mecanismos semelhantes, (3) que uma das infecções favoreça 0 acometimento da outra.

A ocorrência de coinfecções estaria diretamente relacionada ao padrão de circulação desses agentes na população e à prevalência de portadores do AgHBs. Considerando que a prevalência de infecção passada por um dos tipos de plasmódio foi relativamente menor entre os indivíduos de até quinze anos de idade, onde 0 VHB circula com relativa frequêencia, o risco de desenvolver coinfecções não é homogêneo entre a população portadora do AgHBs.

Como 0 VHB não foi encontrado em menores de um ano e os anticorpos contra antígenos do Plasmodium vivax ou Plasmodium falciparum na população estudada estiveram presentes em todas as faixas de idade, aliado ao fato da conhecida pouca importância da transmissão vertical do VHB na regiãa ${ }^{61219}$, levantamos a ideia de que talvez o primeiro contato seja com 0 plasmódio e que a maioria dos indivíduos AgHBs, em risco de desenvolver coinfecção, seja indivíduos com mais de 15 anos de idade, na maioria portadores assintomáticos do VHB. Os eventos provavelmente ocorrem, na grande maioria das vezes, em momentos distintos.

Este estudo demonstra elevada prevalência de malária, moderada endemicidade do VHB e provavelmente baixa ocorrência de coinfecções entre 0 plasmódio e 0 VHB, pelas diferenças apontadas no padrão de distribuição dessas infecções, sendo 0 risco de coinfecção mais importante entre os indivíduos de 15 a 30 anos de idade, apesar da ocorrência de coinfecções no grupo de cinco a 14 anos, onde há transmissão importante do VHB, mostrando padrão heterogêneo da ocorrência de coinfecções, em relação ao espectro clínico da infecção pelo VHB.

\section{REFERÊNCIAS BIBLIOGRÁFICAS}

1. Barcus MJ, Hien TT, White N, Laras K, Farrar J, Schwartz IK, Corwin A, Baird JK. Short Report: Hepatitis B Infection and Severe Plasmodium Falciparum Malaria in Vietnamese Adults. The American Journal of Tropical Medicine and Hygiene 66: 140-142, 2002.

2. Beier JC, Killeen GF, Githure LI. Short report: entomologic inoculation rates and Plasmodium falciparum malaria prevalence in Africa. The American Journal of Tropical Medicine and Hygiene 61: 109-113, 1999.

3. Brabin BJ. Applied field research in malaria reports. World Health Organization, 1991.

4. Brabin L, Brabin BJ. Cultural factors and transmission of hepatitis B virus. American Journal of Epidemiology 122: 725-730, 1985.

5. Brabin L, Brabin BJ, Van der Kaay HJ. High and low spleen rates distinguish two populations of women living under the same malaria endemic conditions in Madang, Papua New Guinea. Transactions of the Royal Society of Tropical Medicine and Hygiene 82: 671-676, 1988.

6. Braga WSM, Melo HO, Cossate MDB, Castilho MC, Souza RAB, Brasil LM, Fonseca JCF. Prevalência de marcadores sorológicos dos vírus da hepatite B e Delta em população assintomática: Estudo do impacto do uso da vacina contra hepatite B em áreas hiperendêmicas, Itamarati- Amazonas, Vale do rio Juruá. Revista da Sociedade Brasileira de Medicina Tropical 31( Supl I) : 31, 1998.

7. Brito JDU. Características clínico-laboratoriais da associação entre a malária humana e a infecção pelo vírus da hepatite B. Tese de Mestrado. Universidade de Brasília, Brasília, 1995.

8. Chanteau S, Sechan Y, Moulia-Pelat JP, Luquiaud P, Spiegel A, Boutin JP, Roux JF. The blackfly Simulium buissoni and infection by hepatitis B virus on a holoendemic island of the Marquesas archipelago in French Polynesia. American Journal of Tropical Medicine and Hygiene 48: 763-770, 1993.

9. Conway DJ, Greenwood BM, McBride JS. Longitudinal study of Plasmodium falciparum polymorphic antigens in a malaria endemic population. Infection and Immunity 60:622-627, 1992.

10. Epi Info, versão 6.04c. Stone Mountain: GA: Inc.; 1998.

11. Focaccia R, Conceição OJG, Sette Jr H, Sabino E, Bassit L, Nitrini DR, Lomar AV, Lourenço R, Souza FV, Kiffer CR, Santos EB, Gonzales MP, Sães-Alquézar A, Riscal JR, Fisher D. Estimated prevalence of viral hepatitis in the general population of the municipality of São Paulo, measured by a serologic survey of a stratified, randomized and residence-based population. The Brazilian Journal of Infectious Diseases 2: 269-284, 1998.

12. Fonseca JCF. Epidemiologia das hepatites B e Delta na região Amazônica. Skopia 23: 28-32, 1988.

13. Fundação de Medicina Tropical do Amazonas. Boletim Trimestral out/dez, 1999. Brasil, 2000.

14. Fundação Nacional de Saúde. Informes da Gerência técnica de Malária. Ministério da Saúde, Brasília, Brasil, 1999.

15. Fundação Nacional de Saúde. Coordenadoria Regional do Amazonas. Informes da Gerência técnica de Malária - AM. Brasil, 1999.

16. Iversson LB, Granato CF, Rosa AT, Pannuti CS. Relationship between the prevalence of antibodies to hepatitis B core antigen and arbovirus in fishermen from the Ribeira Valley, Brazil. Revista do Instituto de Medicina Tropical de São Paulo 32:215-220, 1990.

17. Kiesslich D, Fraiji NA, Crispim MA, Pereira FR, Martinho AC, Campello SC, Almeida TA, Vasquez LS. Prevalência de marcadores sorológicos e moleculares do vírus da hepatite B em gestantes do Estado do Amazonas. Epidemiologia e Serviços de Saúde 12: 155-164, 2003.

18. Mendez M, Arce M, Kruger HP, Sanches SA. Prevalência de marcadores serologicos de hepatitis virica en diversos grupos de poblacion del Peru. Boletin de la Oficina Sanitaria Panamericana 106: 127-138, 1989.

19. Pasquetto V, Guidotti LG, Kakimi K, Tsuji M, Chisari FV. Host-Virus Interactions during Malaria Infection in Hepatitis B virus Transgenic Mice. Journal of Experimental Medicine 192: 529-535, 2000.

20. Scalise G, Mazaheri MR, Kremastinou J, Howard CR, Sorensen K, Zuckerman AJ. Transmission of hepatitis B to the Rhesus monkey. Studies of 
humoral and cell-mediated response. Journal of Medical Primatology 7: 114-118, 1978

21. Souto FJD. Distribuição da hepatite B no Brasil: Atualização do mapa epidemiológico e proposições para seu controle. Revista da Sociedade Brasileira de Gastroenterologia 18: 143-150, 1999.
22. Thursz MR, Kwiatkowski D, Torok ME, Allsopp CE, Greenwood BM, Whittle $\mathrm{HC}$, Thomas HC, Hill AV. Association of hepatitis B surface antigen carriage with severe malaria in Gambian children. Nature Medicine 1: 374-375, 1995.

23. Voller A, Draper MA. Immunodiagnosis and seroepidemiology of malaria. British Medical Bulletin 38: 173-177, 1982. 